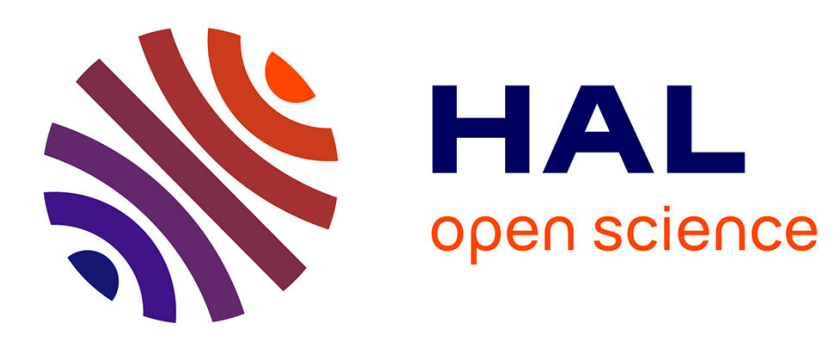

\title{
Axisymmetric deformations of a 2nd grade elastic cylinder
}

\author{
I. Giorgio, F Dell'Isola, D J Steigmann
}

\section{To cite this version:}

I. Giorgio, F Dell'Isola, D J Steigmann. Axisymmetric deformations of a 2nd grade elastic cylinder. 2018. hal-01870649v2

\section{HAL Id: hal-01870649 \\ https://hal.science/hal-01870649v2}

Preprint submitted on 18 Sep 2018

HAL is a multi-disciplinary open access archive for the deposit and dissemination of scientific research documents, whether they are published or not. The documents may come from teaching and research institutions in France or abroad, or from public or private research centers.
L'archive ouverte pluridisciplinaire HAL, est destinée au dépôt et à la diffusion de documents scientifiques de niveau recherche, publiés ou non, émanant des établissements d'enseignement et de recherche français ou étrangers, des laboratoires publics ou privés. 


\title{
Axisymmetric deformations of a 2nd grade elastic cylinder
}

\author{
I. Giorgio ${ }^{\mathrm{a}, \mathrm{c}, *}$, F. dell'Isola ${ }^{\mathrm{a}, \mathrm{c}}$, D. J. Steigmann ${ }^{\mathrm{b}, \mathrm{c}}$ \\ ${ }^{a}$ Dipartimento di Ingegneria Strutturale e Geotecnica, \\ Università di Roma La Sapienza, Roma, Italy \\ ${ }^{b}$ Department of Mechanical Engineering \\ University of California, Berkeley, CA 94720, USA \\ ${ }^{c}$ International Centre for the Mathematics and Mechanics of Complex Systems \\ Università dell'Aquila, L'Aquila, Italy
}

\begin{abstract}
Numerical simulations of the axisymmetric response of a 2nd grade, fiber-reinforced, elastic cylinder are presented and discussed. The underlying model accounts for surface strain and for bending and twist, as in conventional theories, and also includes the non-standard effect of geodesic fiber bending at the constitutive level.
\end{abstract}

Keywords: Woven fabrics, elastic surface theory, strain gradients, elastic cylinder

\section{Introduction}

Our purpose in this short communication is to illustrate the equilibrium response of 2nd grade elastic cylinders in axial tension and compression. The cylinder is regarded as a surface composed of intersecting fibers that offer elastic resistance to stretching, flexure and twist. Conventional plate and shell theories account for elastic resistance of the surface to strain, twist and normal bending. In the present model, discussed comprehensively in [1], a non-standard elastic resistance to geodesic bending is also taken into account (see also [2]). This latter mode of bending is the source of a strain-gradient effect, which is not present in the constitutive equations of conventional theories of plates and shells but which is nevertheless non-negligible in fiber sheets. The general topic is the subject of extensive current research on pantographic sheets. We refer to [3] for a comprehensive review and bibliography.

We assume the surface to be in a natural, relaxed state in a plane configuration wherein the fibers are orthogonal, straight and untwisted. The surface is then mapped to a right circular cylinder capped by rigid rings at its ends, and subsequently extended or compressed in the presence of various boundary conditions. The predicted deformations are obtained using the commercial software package COMSOL Multiphysics ${ }^{T M}$. This code furnishes a particularly convenient platform for our purpose. In particular, it requires as input only the explicit expression for the strain-energy function. The program then constructs an associated weak form of the relevant equilibrium equations together with its finite-element implementation. Applications of the finite element method specific to second-gradient or micromorphic elasticity $[4,5,6,7,8,9]$ are discussed in $[10,11,12,13,14,15,16]$.

In Section 2 we recall the formulation introduced in [1] for the strain-energy function together with the underlying kinematical framework. This is reduced, in Section 3 , to a one-dimensional model for axisymmetric deformations, with the axial coordinate along the reference cylinder playing the role of the single space dimension. Section 4 concludes with a presentation and discussion of

${ }^{*}$ Corresponding author 
numerical examples.

\section{Strain-energy function, axisymmetry}

Our objective is to determine the position field $\mathbf{r}\left(u_{1}, u_{2}\right)$ of the surface in equilibrium, where $u_{\alpha}$ are the Cartesian coordinates of a material point on a plane. These are taken to be $u_{1}=z$ and $u_{2}=R \theta$, where $z \in[-L, L]$ is the axial coordinate on the right circular cylinder, of radius $R$, onto which the plane is mapped, and $\theta \in[-\pi, \pi]$ is the azimuthal angle on the cylinder. Thus the plane configuration of the sheet is a rectangle of length $2 L$ and width $2 \pi R$.

The model is based on the strain-energy function $[1$, $17]$

$$
\begin{aligned}
W= & w\left(\epsilon_{L}, \epsilon_{M}, J\right)+\frac{1}{2} A_{L}\left|L_{\alpha} L_{\beta} \mathbf{r}_{, \alpha \beta}\right|^{2}+ \\
& \frac{1}{2} A_{M}\left|M_{\alpha} M_{\beta} \mathbf{r}_{, \alpha \beta}\right|^{2}+\frac{1}{2} A_{\Gamma}\left|L_{\alpha} M_{\beta} \mathbf{r}_{, \alpha \beta}\right|^{2},
\end{aligned}
$$

where $A_{L}, A_{M}, A_{\Gamma}$ are material constants, $L_{\alpha}$ and $M_{\alpha}$ $(\alpha=1,2)$ are the Cartesian components of the unit tangent vectors to the orthogonal fibers on the plane; and

$$
\begin{aligned}
\epsilon_{L} & =E_{\alpha \beta} L_{\alpha} L_{\beta}, \quad \epsilon_{M}=E_{\alpha \beta} M_{\alpha} M_{\beta}, \\
J & =\left|L_{\alpha} M_{\beta} \mathbf{r}_{, \alpha} \times \mathbf{r}_{, \beta}\right|
\end{aligned}
$$

are the extensional fiber strains and the areal dilation, where

$$
E_{\alpha \beta}=\frac{1}{2}\left(\mathbf{r}_{, \alpha} \cdot \mathbf{r}_{, \beta}-\delta_{\alpha \beta}\right),
$$

in which $\delta_{\alpha \beta}$ is the Kronecker delta, is the Lagrange strain. Here and elsewhere, $\mathbf{r}_{, \alpha}=\partial \mathbf{r} / \partial u_{\alpha}$ and $\mathbf{r}_{, \alpha \beta}=$ $\partial^{2} \mathbf{r} / \partial u_{\alpha} \partial u_{\beta}$.

The second and third terms in Eq. (1) attribute energy to the fiber stretches and their gradients in the directions of the fibers, and to the normal and geodesic bending of the fibers. The fourth term accounts for twist of the fibers as the surface deforms, and for the cross derivatives of the fiber stretches in directions orthogonal to the fibers. The latter derivatives induce stretch along parallel fibers in a small neighborhood of a material point. We refer the interested reader to $[1,17]$ for a full discussion of the physical significance of the various terms represented in Eq. (1).

For the strain-dependent term in Eq. (1) we assume $[1,17]$

$$
w\left(\epsilon_{L}, \epsilon_{M}, J\right)=\frac{1}{2}\left(E_{L} \epsilon_{L}^{2}+E_{M} \epsilon_{M}^{2}\right)-G(\ln J+1-J),
$$

where $E_{L, M}$ and $G$ are positive constants. This energy does not include a term proportional to $\epsilon_{L} \epsilon_{M}$ and therefore does not exhibit a Poisson effect with respect to the fiber axes. The term involving $J$ penalizes fiber collapse $(J \rightarrow 0)$ by imposing unbounded growth of the energy, whereas the remaining terms are appropriate for smallto-moderate fiber strains.

In this work we assume the fibers to be straight on the reference plane and oriented at $\pm 45^{\circ}$ to the edges of the rectangle, with

$$
L_{1}=L_{2}=\frac{\sqrt{2}}{2} \quad \text { and } \quad M_{1}=-M_{2}=-\frac{\sqrt{2}}{2} .
$$

We seek axisymmetric deformations that map the cylinder to the surface of revolution described by

$$
\mathbf{r}=r(z) \mathbf{e}_{r}(\theta)+\xi(z) \mathbf{k},
$$

where $r$ and $\xi$ are the radial and axial coordinates of the circle $z=$ const. of material points on the cylinder, $\mathbf{k}$ is a unit vector aligned with the axis of the cylinder and $\mathbf{e}_{r}(\theta)$ is the radial unit vector in the cross section of the cylinder at azimuth $\theta$.

\section{One-dimensional formulation}

A straightforward calculation furnishes

$$
\mathbf{r}_{, 1}=r^{\prime} \mathbf{e}_{r}+\xi^{\prime} \mathbf{k}, \quad \mathbf{r}_{, 2}=\frac{r}{R} \mathbf{e}_{\theta}
$$

and

$$
\begin{aligned}
& \mathbf{r}_{, 11}=r^{\prime \prime} \mathbf{e}_{r}+\xi^{\prime \prime} \mathbf{k}, \quad \mathbf{r}_{, 22}=-\frac{r}{R^{2}} \mathbf{e}_{r}, \\
& \mathbf{r}_{, 12}=\mathbf{r}_{, 21}=\frac{r^{\prime}}{R} \mathbf{e}_{\theta},
\end{aligned}
$$


where $(\cdot)^{\prime}=d(\cdot) / d z$ and $\mathbf{e}_{\theta}=\mathbf{k} \times \mathbf{e}_{r}$ is the azimuthal unit vector in a cross-sectional plane.

From these we obtain the fiber strains

$$
\epsilon_{L}=\epsilon_{M}=\frac{1}{2}\left(E_{11}+E_{22}\right)
$$

where

$$
E_{11}=\frac{1}{2}\left[\left(r^{\prime}\right)^{2}+\left(\xi^{\prime}\right)^{2}-1\right] \text { and } E_{22}=\frac{1}{2}\left[\left(\frac{r}{R}\right)^{2}-1\right],
$$

whereas $E_{12}=0$, and the surface dilation

$$
J=\frac{r}{R}\left[\left(r^{\prime}\right)^{2}+\left(\xi^{\prime}\right)^{2}\right]^{1 / 2}
$$

together with

$$
\begin{aligned}
&\left|L_{\alpha} L_{\beta} \mathbf{r}_{, \alpha \beta}\right|^{2}=\left|M_{\alpha} M_{\beta} \mathbf{r}_{, \alpha \beta}\right|^{2}= \\
& \frac{1}{4}\left[\left(r^{\prime \prime}-\frac{r}{R^{2}}\right)^{2}+4\left(\frac{r^{\prime}}{R}\right)^{2}+\left(\xi^{\prime \prime}\right)^{2}\right]
\end{aligned}
$$

and

$$
\left|L_{\alpha} M_{\beta} \mathbf{r}_{, \alpha \beta}\right|^{2}=\frac{1}{4}\left[\left(r^{\prime \prime}+\frac{r}{R^{2}}\right)^{2}+\left(\xi^{\prime \prime}\right)^{2}\right] .
$$

Substitution into Eq. (1) yields a strain-energy function of the form

$$
W=W\left(r, r^{\prime}, r^{\prime \prime}, \xi^{\prime}, \xi^{\prime \prime}\right)
$$

\section{Examples}

All examples pertain to a cylinder of radius $R=1$ cm and length $L=10 \mathrm{~cm}$. We assume the function $\xi(z)$ to be odd $(\xi(-z)=-\xi(z))$ and the function $r(z)$ to be even $(r(-z)=r(z))$, and thus model the half-cylinder $z=[0, L]$ with $\xi(0)=0$ and $r^{\prime}(0)=0$. The elastic moduli are $E_{L}=E_{M}=1 \times 10^{7} \mathrm{~N} / \mathrm{m}$ and $G=0.2 \times 10^{7}$ $\mathrm{N} / \mathrm{m}$. In the 2nd gradient terms in Eq. (1), the moduli are $A_{L}=A_{M}=100 \mathrm{Nm}$ and $A_{\Gamma}=20 \mathrm{Nm}$. The one-dimensional domain is discretized using 250 finite elements with Hermite polynomials of quintic order, and a sequence of axial displacements is imposed at $z=L$ up to a maximum absolute value of $3 \mathrm{~cm}$. We note that $C^{1}$ continuous cubic Hermite polynomials are sufficient for our purposes, but quintic Hermite polynomials provide improved accuracy using the same spacial grid.

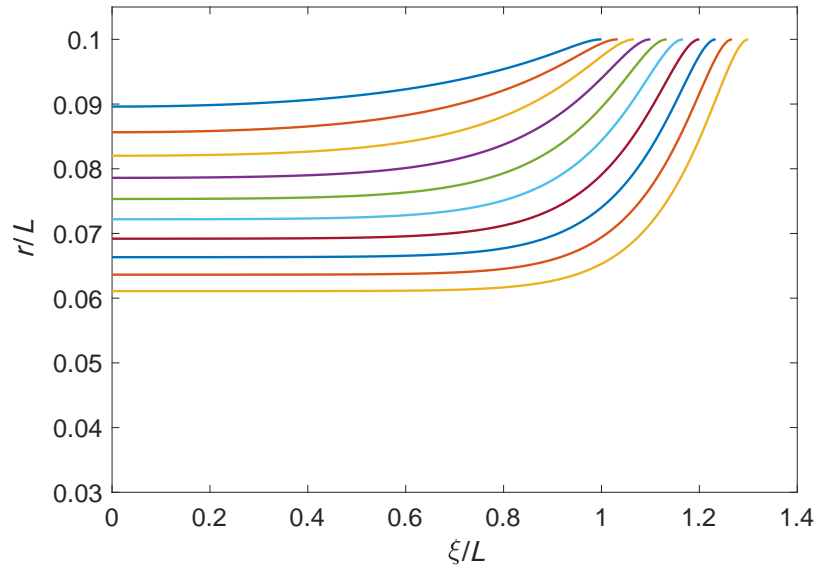

Figure 1: Tension test with fully clamped boundary conditions. Each color refers to a different imposed axial displacement.

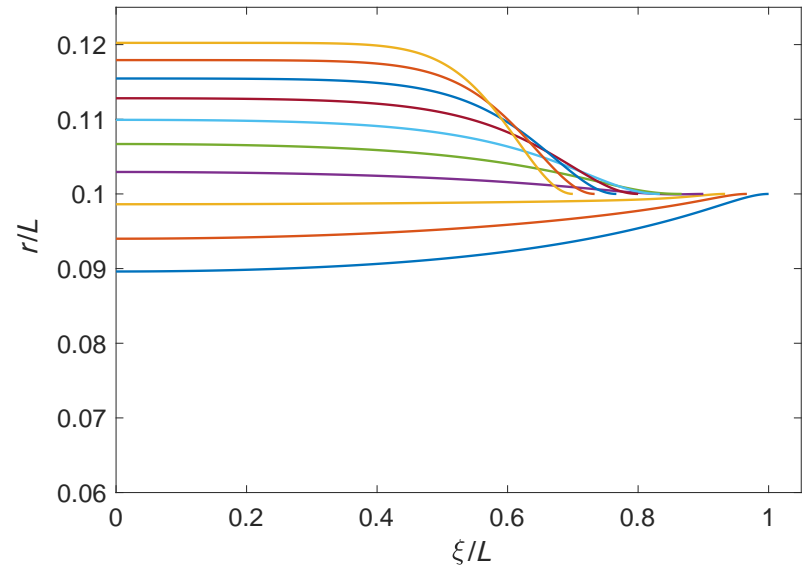

Figure 2: Compression test with fully clamped boundary conditions. Each color refers to a different imposed axial displacement.

We consider two basic examples. In each of these we impose $r(L)=R$ and $r^{\prime}(L)=0$ together with various values of the deformed axial length $l=\xi(L)$. In the first class of examples we impose $\xi^{\prime}(L)=1$. We refer to this as a fully clamped boundary. This can be interpreted as a short rigid collar of length $h$ and radius $R$ glued to the end of the cylinder. A Taylor expansion furnishes

$$
\xi(L+h)-\xi(L)=h \xi^{\prime}(L)+o(h),
$$


whereas the rigidity of the collar implies that $\xi(L+h)-$ $\xi(L)=h$. Our boundary condition provides the leadingorder approximation to this condition for small $h$. Similar reasoning justifies the boundary condition $r^{\prime}(L)=0$. This ensures that $r(L+h)=r(L)$ at leading order.

Figures 1 and 2 depict the deformed meridians in equilibrium; i.e., plots of normalized $r$ vs. $\xi$, corresponding to imposed axial extension or compression.

These exhibit a local effect associated with the boundary conditions, while in the interior of the sheet the radius approaches a nearly uniform value. This stands in contrast to the classical necking response of a pure membrane model in tension, in which the membrane radius decreases monotonically as the symmetry plane $z=0$ is approached. However, pure membrane theory is not appropriate in the present circumstances as it does not account for the higher-gradient effects associated with fiber bending and twisting resistance. For small compressive axial displacement, we predict a reduction in radius relative to its reference value. The radius is seen to increase as the axial displacement is increased. Presumably this transition to a deformation mode in which the radius is larger and the fiber curvatures are smaller serves to lower the overall energy and may be associated with post-buckling response. This conclusion is not definitive, however, as we have not conducted an analysis of potential bifurcation.

A perspective view of the compressed cylinder, showing the deformed fiber trajectories, is provided in Figure 3. In the practical example of standard cable sleeving, we observe a similar bulging of cylinders composed of a mesh of woven fibers [18] into surfaces of revolution having nearly uniform radius over most of their length. Such deformation modes are also characteristic of $\mathrm{McK}$ ibben artificial muscles [19].

In the second class of examples we relax the boundary condition $\xi^{\prime}(L)=1$. In the weak form of the equilibrium equations, the test function associated with $\xi^{\prime}(L)$ is workconjugate to a double force $W_{\xi^{\prime \prime}}$ acting at $z=L[20]$. Relaxation of the restriction on $\xi^{\prime}(L)$ therefore ensures the vanishing of the double force in the weak sense. In general the double force may be interpreted as a pair of equal and opposite forces separated by the small distance $h$ that act to maintain the relative separation $\xi(L+h)-\xi(L)$. A vanishing double force means that no agency is operating to control this separation. Physically, this corresponds to insertion of the end of the cylinder, as before, into a rigid collar of radius $R$. The collar acts as a sleeve and the material of the cylinder is free to slide without friction inside it.

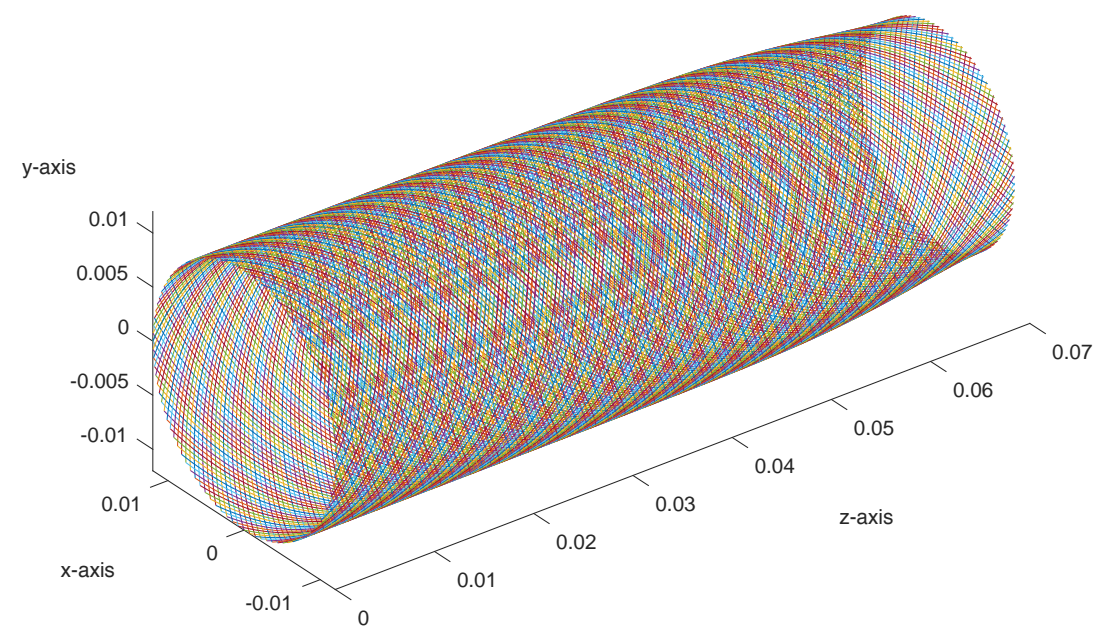

Figure 3: Perspective view of the compressed cylinder. Lengths are normalized with respect to $L$. 


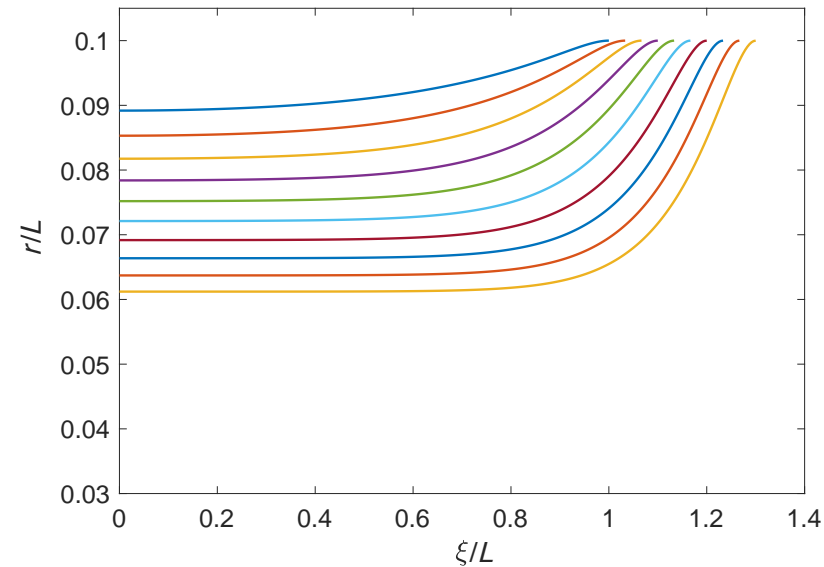

Figure 4: Tension test with zero double force. Each color refers to a different imposed axial displacement.

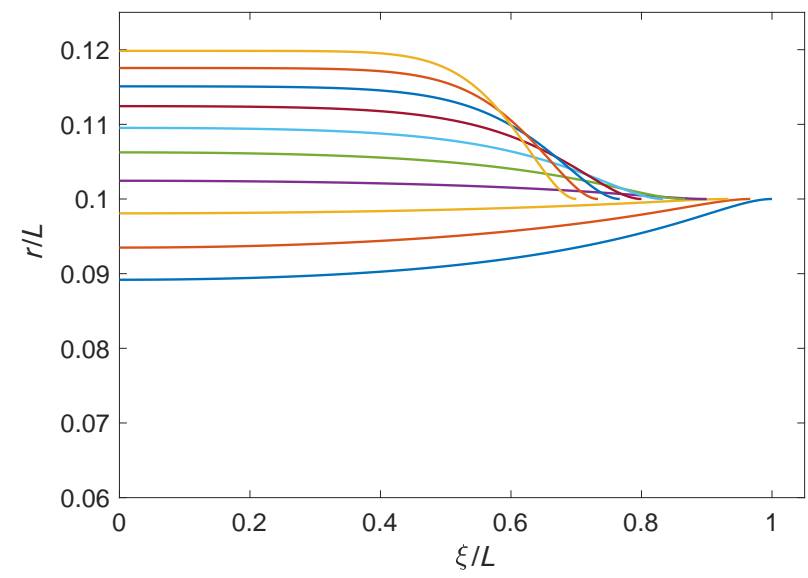

Figure 5: Compression test with zero double force. Each color refers to a different imposed axial displacement.

Figures 4 and 5 depict the deformed surface under tension and compression with zero double force. The response is very similar to that of the fully clamped cylinder, but minor quantitative differences are observable.

In general the double force is a vector $\boldsymbol{\mu}[20]$ which is given, in the present circumstances, by

$$
\boldsymbol{\mu}=W_{r^{\prime \prime}} \mathbf{e}_{r}+W_{\xi^{\prime \prime}} \mathbf{k}
$$

This is turn generates the edge couple $\mathbf{c}$, per unit length of the edge $z=L$, given by $\mathbf{c}=\mathbf{r}^{\prime} \times \boldsymbol{\mu}[20]$. Thus,

$$
\mathbf{c}=\left(\xi^{\prime} W_{r^{\prime \prime}}-r^{\prime} W_{\xi^{\prime \prime}}\right) \mathbf{e}_{\theta},
$$

which reduces to the familiar bending moment

$$
\mathbf{c}=W_{r^{\prime \prime}} \mathbf{e}_{\theta}
$$

in the case of a fully clamped boundary.

Acknowledgments: The work of DJS is supported by the US National Science Foundation through grant number CMMI-1538228.

\section{References}

[1] I. Giorgio, R. Grygoruk, F. dell'Isola, D. J. Steigmann, Pattern formation in the threedimensional deformations of fibered sheets, Mechanics Research Communications 69 (2015) 164-171.

[2] A. Misra, T. Lekszycki, I. Giorgio, G. Ganzosch, W. H. Müller, F. dell'Isola, Pantographic metamaterials show atypical Poynting effect reversal, Mechanics Research Communications 89 (2018) 6-10.

[3] F. dell'Isola, P. Seppecher, et al., Pantographic metamaterials: an example of mathematically driven design and of its technological challenges, Continuum Mechanics and Thermodynamics (2018) 1-34doi:10.1007/s00161-018-0689-8.

[4] E. Barchiesi, M. Spagnuolo, L. Placidi, Mechanical metamaterials: a state of the art, Mathematics and Mechanics of Solidsdoi:10.1177/ 1081286517735695.

[5] C. I. Kim, M. Zeidi, Gradient elasticity theory for fiber composites with fibers resistant to extension and flexure, International Journal of Engineering Sciencedoi: 10.1016/j.ijengsci.2018.06.002.

[6] J. Altenbach, H. Altenbach, V. A. Eremeyev, On generalized Cosserat-type theories of plates and shells: a short review and bibliography, Archive of Applied Mechanics 80 (1) (2010) 73-92. 
[7] S. Khakalo, V. Balobanov, J. Niiranen, Modelling size-dependent bending, buckling and vibrations of $2 \mathrm{~d}$ triangular lattices by strain gradient elasticity models: applications to sandwich beams and auxetics, International Journal of Engineering Science 127 (2018) 33-52.

[8] B. E. Abali, C. Völlmecke, B. Woodward, M. Kashtalyan, I. Guz, W. H. Müller, Three-dimensional elastic deformation of functionally graded isotropic plates under point loading, Composite Structures 118 (2014) 367-376.

[9] A. Khludnev, G. R. Leugering, Delaminated thin elastic inclusions inside elastic bodies, Mathematics and mechanics of complex systems 2 (1) (2013) 1-21.

[10] F. Maurin, F. Greco, W. Desmet, Isogeometric analysis for nonlinear planar pantographic lattice: discrete and continuum models, Continuum Mechanics and Thermodynamics (2018) 1-14doi:10.1007/ s00161-018-0641-y.

[11] L. Greco, M. Cuomo, L. Contrafatto, A reconstructed local $\bar{B}$ formulation for isogeometric Kirchhoff-Love shells, Computer Methods in Applied Mechanics and Engineering 332 (2018) 462487.

[12] L. Greco, M. Cuomo, L. Contrafatto, S. Gazzo, An efficient blended mixed B-spline formulation for removing membrane locking in plane curved Kirchhoff rods, Computer Methods in Applied Mechanics and Engineering 324 (2017) 476-511.

[13] V. Balobanov, J. Niiranen, Locking-free variational formulations and isogeometric analysis for the Timoshenko beam models of strain gradient and classical elasticity, Computer Methods in Applied Mechanics and Engineering 339 (2018) 137-159.

[14] A. Cazzani, M. Malagù, E. Turco, F. Stochino, Constitutive models for strongly curved beams in the frame of isogeometric analysis, Mathematics and Mechanics of Solids 21 (2) (2016) 182-209.

[15] J. Niiranen, V. Balobanov, J. Kiendl, S. B. Hosseini, Variational formulations, model comparisons and numerical methods for Euler-Bernoulli microand nano-beam models, Mathematics and Mechanics of Solidsdoi : 10.1177/1081286517739669.

[16] S. Khakalo, J. Niiranen, Isogeometric analysis of higher-order gradient elasticity by user elements of a commercial finite element software, ComputerAided Design 82 (2017) 154-169.

[17] I. Giorgio, A. Della Corte, F. dell'Isola, D. J. Steigmann, Buckling modes in pantographic lattices, Comptes rendus Mecanique 344 (7) (2016) 487-501.

[18] https://www.mcmaster.com [online].

[19] M. G. Antonelli, P. Beomonte Zobel, F. Durante, T. Raparelli, Numerical modelling and experimental validation of a McKibben pneumatic muscle actuator, Journal of Intelligent Material Systems and Structures 28 (19) (2017) 2737-2748.

[20] D. J. Steigmann, Equilibrium of elastic lattice shells, Journal of Engineering Mathematics 109 (1) (2018) $47-61$. 